\title{
BK Virus Screening and Management Following Kidney Transplantation: An Update
}

\author{
Phuong-Thu Pham ${ }^{1 *}$, Joanna Schaenman² and Phuong-Chi Pham ${ }^{3}$ \\ ${ }^{1}$ Department of Medicine, Nephrology Division, David Geffen School of Medicine at UCLA, Los Angeles, CA 90095, USA \\ ${ }^{2}$ Department of Medicine, Division of Infectious Diseases, David Geffen School of Medicine at UCLA, Los Angeles, CA 90095, USA \\ ${ }^{3}$ Department of Medicine, Nephrology and Hypertension Division, UCLA-Olive View Medical Center, Sylmar, CA 91342, USA
}

\begin{abstract}
BK virus is a ubiquitous human virus with a peak incidence of primary infection in children $2-5$ years of age and a seroprevalence rate of greater than $60-90 \%$ among the adult population worldwide. Following primary infection, BK virus preferentially establishes latency within the genitourinary tract and frequently reactivates in the setting of immunosuppression. In renal transplant recipients, BK virus is associated with a range of clinical syndromes including asymptomatic viruria with or without viremia, ureteral stenosis and obstruction, interstitial nephritis, and BK allograft nephropathy (BKN). BKN most commonly presents with an asymptomatic rise in serum creatinine between 2 to 60 months after engraftment (median 9 months). A definitive diagnosis requires an allograft biopsy. Over the last two decades, BKN has been recognized as an important cause of allograft dysfunction and graft loss in kidney transplant recipients. Nonetheless, there is currently no standardized protocol for the management of BK viremia or established $\mathrm{BKN}$. In this article, a brief overview of the literature on the various treatment strategies for BK-associated clinical spectrum is presented followed by the authors' suggested approach for posttransplant screening and monitoring for BK virus replication. Suggested treatment strategies are also discussed.
\end{abstract}

Keywords: BKV screening and management; Kidney transplantation; $\mathrm{BKV}$ viuria; BKV viremia; BK nephropathy; Antiviral therapy in BK associated clinical syndromes; Intravenous immunoglobulin (IVIG)

\section{Literature Overview}

Although treatment strategies for BK viremia with or without BK nephropathy vary widely among centers, immunological containment of BK virus replication has been accepted as the mainstay of therapy. Suggested strategies for reduction in immunosuppression include reduction or discontinuation of antimetabolites (mycophenolate mofetil or azathioprine), reduction in calcineurin inhibitor therapy, or immunosuppressant class switch.

Registry analyses and prospective randomized clinical trials suggested that tacrolimus is associated with a higher risk of BK reactivation than cyclosporine (CSA) [1-3]. In an analysis of the Organ Procurement Transplantation Network (OPTN) database consisting of more than 48,000 primary kidney transplant recipients, the cumulative incidence of treatment of BK infection within the first 2 years posttransplant was shown to be significantly higher in patients treated with tacrolimus than those receiving CSA maintenance immunosuppression at discharge $(4.0 \%$ vs. $1.7 \%$, respectively, $\mathrm{p}<0.001)$ [1]. The same study also demonstrated a lower incidence of BK treatment in patients who did not receive antimetabolite at discharge compared with those who received mycophenolate mofetil (MMF) at discharge. Similar to registry data, results of the DIRECT study (in which de novo transplant recipients were randomized to receive either CSA or tacrolimus) revealed a lower incidence of BK viremia in CSAtreated patients compared with their tacrolimus-treated counterparts at 1 -year ( $4.8 \%$ vs. $12.1 \%, \mathrm{p}=0.004$ ) [3]. In vitro studies suggest that CSA may exert an antiviral effect on BKV via suppression of viral replication $[4,5]$ whereas tacrolimus has been reported to activate BKV replication in primary human tubular epithelial cells via the FK-binding protein 12 pathway [6]. Nonetheless, not all studies demonstrated a lower incidence of BK viremia with tacrolimus compared with CSA-treated patients [7]. Single-center studies suggest that tacrolimus and MMF combination therapy result in a significantly higher incidence of BK viuria/BKN compared with other regimens. It is hypothesized that tacrolimus-MMF create a permissive immunosuppressive environment for BKV replication [8].
Early anecdotal case reports demonstrated that tacrolimus to cyclosporine or sirolimus conversion therapy resulted in resolution of BKN and viremia/viuria. Recent experimental animal models have shown that mTOR inhibition may promote differentiation of antiviral memory CD8 T-lymphocytes [9] and enhance their magnitude and quality [10]. Analysis of the OPTN registry database demonstrated a lower 2-year cumulative incidence of "treatment for BK" (as reported on the kidney follow-up forms) in primary kidney transplant recipients receiving mTOR inhibitor therapy at hospital discharge $(n=5380)$ when compared with patients on other regimens without mTOR inhibitor $(\mathrm{n}=42912,1.74 \%$ vs. $3.67 \%$, respectively, $\mathrm{p}<0.001)$ [1].

In BKV-associated disease refractory to reduction in immunosuppressive therapy alone, antiviral agents such as cidofovir, leflunomide, intravenous immunoglobulins (IVIG) [11] and fluoroquinolones have been used in anecdotal case reports and small series of patients with variable success. Nonetheless, systemic review of 40 studies ( 3 randomized controlled trials, 2 prospective cohort, 6 retrospective cohort, and 29 case series studies) showed no graft survival benefit of adding cidofovir or leflunomide to immunosuppressive reduction for the management of BKN [12]. Experimental studies suggest that human intravenous immune globulin (IVIG) preparations contain BKV neutralizing antibodies [13]. However, the role of these antibodies in clearing viral infection remains to be explored. In a single-center study consisting of 70 renal transplant recipients with BKV infection, Bohl et al. [14] showed that while there was a strong

*Corresponding author: Phuong-Thu Pham, Department of Medicine, Nephrology Division, Kidney and Pancreas Transplant Program, David Geffen School of Medicine at UCLA, Los Angeles, CA 90095, USA, Tel: (310) 794-1757; E-mail: PPham@mednet.ucla.edu

Received November 26, 2012; Accepted November 30, 2012; Published December 03, 2012

Citation: Pham PT, Schaenman J, Pham PC (2012) BK Virus Screening and Management Following Kidney Transplantation: An Update. J Transplant Technol Res 2: e119. doi:10.4172/2161-0991.1000e119

Copyright: $\odot 2012$ Pham PT, et al. This is an open-access article distributed under the terms of the Creative Commons Attribution License, which permits unrestricted use, distribution, and reproduction in any medium, provided the original author and source are credited. 
relationship between the intensity of BKV infection and IgG BKVspecific antibody titers, no association between antibody response and viral clearance was observed. It is speculated that humoral response offers incomplete protection and adequate control of BK involves virus-specific cellular immune response. The detection of interferon- $\gamma$ secretion from $B K$ virus-specific $T$ cells has been shown to be associated with resolution of BK viremia [15]. Fluoroquinolones have been shown to inhibit BK DNA topoisomerase and SV40 large tumor antigen (T antigen) helicase. Limited data suggest that fluoroquinolones are effective at preventing BK viremia after renal transplantation [16] .The cidofovir lipid conjugate CMX001 (currently under development for CMV prophylaxis) has been shown in in vitro studies to have anti-polyomavirus activity. Its use in recipients of hematopoietic cell transplant has shown promise for mitigation of BK virus-associated complications [17]. Whether CMX001 might prove to be effective in the treatment of BKV-associated clinical syndromes awaits studies.
Early reports showed a $30 \%$ to greater than $60 \%$ progressive decline in renal function and graft loss in patients with established BKN despite various treatment strategies. More recent studies demonstrated that early diagnosis and intervention may improve prognosis. In a retrospective 5-year review of an open-label, prospective trial of renal transplant recipients randomized to receive either tacrolimus or CSA, minimization of immunosuppression upon detection of viremia was found to be associated with excellent 5-year graft survival (84\%), low rejection rates (12\%), and excellent allograft function (eGFR $63 \mathrm{~mL} /$ min for tacrolimus- and $52 \mathrm{~mL} / \mathrm{min}$ for cyclosporine-treated patients) [7]. In the study, antimetabolite was withdrawn upon detection of BK viremia, and calcineurin inhibitor minimized in cases of sustained viremia. There was no difference in overall graft loss between patients with and without viremia or sustained viremia. Furthermore, there was no evidence of clinical BKN at 5 year follow-up.

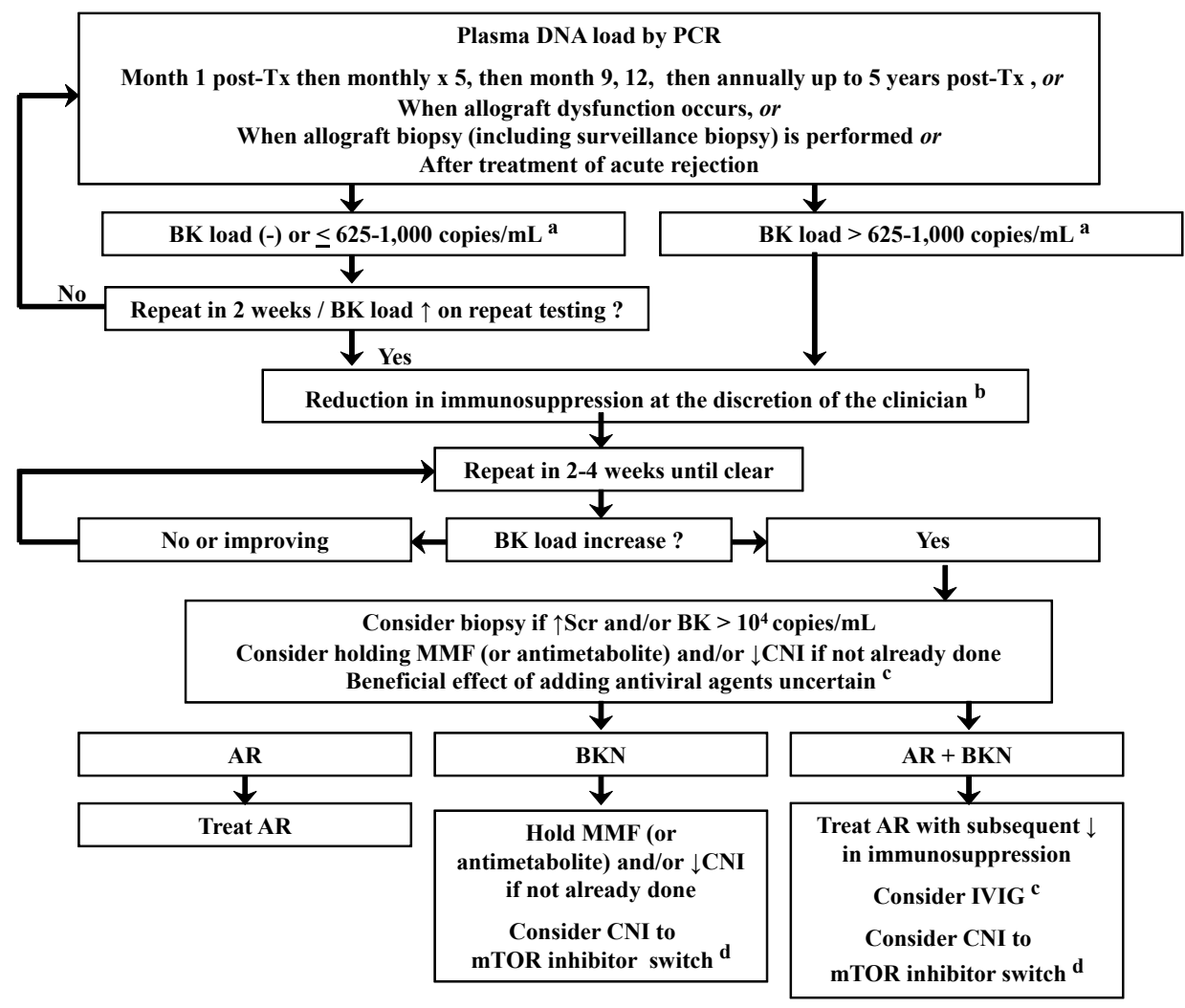

\footnotetext{
${ }^{a}$ No standardized PCR assays for BKV are currently available. Cut-off levels for viral detection should be based on PCR assays used at individual institutions

${ }^{b}$ Common practice: 1) $\downarrow$ or Hold MMF (or antimetabolite) 2) $\downarrow$ (MMF + CNI) by 25-50\% 3) $\downarrow$ CNI . Less common practice: 1) $\downarrow$ Prednisone $+/-\downarrow$ CNI 2) Tacrolimus to CSA switch 3) CNI to mTOR inhibitor switch $+/-\downarrow$ MMF (ongoing clinical trials)

c See text

d Evidence-based recommendations are lacking. May avoid long-term nephrotoxic effect of CNI therapy. Not recommended in patients with baseline significant proteinuria (arbitrarily defined as $\geq 500 \mathrm{mg} / \mathbf{2 4 h r}$ )
}

Abbreviations: Post-Tx: post-transplant; SCr: serum creatinine; MMF: mycophenolate mofetil; CNI: calcineurin inhibitor; AR: acute rejection; BKN: BK nephropathy; mTOR: mammalian target of rapamycin; IVIG: intravenous immunoglobulins

Figure 1: Suggested approach for screening \& management of BKV-associated clinical syndromes. 


\section{Treatment Strategies for BK-Associated Clinical Syndromes: The KDOQI Guidelines and the Authors' Perspective}

\section{The KDOQI guidelines}

The 2009 KDIGO clinical practice guidelines suggested screening all renal transplant recipients for BKV with quantitative plasma NAT (nucleic acid testing) at least monthly for the first 3-6 months after transplantation, then every 3 months until the end of the first post transplant year, whenever there is an unexplained rise in serum creatinine, and after treatment for acute rejection [18]. The guidelines suggest reducing immunosuppressive medications when BKV plasma NAT is persistently greater than 10,000 copies $/ \mathrm{mL}$.

\section{The authors' perspective}

Owing to the lack of evidence-based treatment options targeting viral replication and high rates of graft loss in established BKN, intensive monitoring of urine and/or serum for BK by polymerase chain reaction (PCR) and judicious reduction of immunosuppressive therapy is warranted. A proactive, preemptive approach often leads to resolution of viuria and/or viremia and halts the progression of BK viremia to BK nephropathy. Studies have demonstrated that the highest prevalence of BK viuria and viremia occurs at 2-3 months, and 3-6 months, respectively. Hence, it seems plausible to monitor for BK viruria and/or viremia at least monthly for the first 3-6 months after transplantation. Steroid pulses used to treat rejection without reduction in maintenance immunosuppression have been shown to be associated with an increased incidence of BK viuria, viremia and nephropathy [19]. Analyses of US transplant registry database also demonstrated an increase in the incidence of "treatment for BK (polyoma) virus" (as reported on the kidney follow-up forms) in patients receiving induction therapy with rabbit antithymocyte globulin (Thymoglobulin) compared with those receiving IL-2 receptor antagonist induction $[1,20]$. Hence, it would be reasonable to screen patients for BKV after treatment for acute rejection particularly when T-lymphocyte depleting agent is used.

While the KDOQI guidelines recommend reduction in immunosuppression when BKV plasma NAT is persistently greater than $10^{4}$ copies $/ \mathrm{mL}$, in the authors' opinion rising plasma BKV DNA copies on serial measurements also warrants immunosuppression reduction irrespective of BKV copy levels. Although monitoring for urine or plasma BK or both is largely institution dependent, monitoring for plasma BK alone appears to be cost effective because BKN is unusual in the absence of BK viremia. Patients with biopsy-proven BKN and concurrent acute rejection should be treated with antirejection treatment, with subsequent reduction in immunosuppression at the discretion of the clinicians. Anecdotal case reports suggested that IVIG may be effective in treating steroid-resistant rejection and its use may be beneficial in patients with concomitant rejection and BKN or in those with histopathological changes that are indistinguishable from those of rejection [21,22].

Although tacrolimus and MMF combination therapy has been shown to result in a significantly higher incidence of BK viuria/BKN compared with cyclosporine-MMF therapy, further studies are needed before tacrolimus to CSA switch for BK viremia/BKN can be routinely recommended. It is noteworthy that mycophenolic acid concentrations are lower in patients receiving CSA-based immunosuppression compared to those treated with tacrolimus containing regimen. It is speculated that tacrolimus-MMF combination therapy can lead to higher overall degree of immunosuppression and increased $\mathrm{BK}$ replication risk. While MMF dosing was significantly lower in patients randomized to tacrolimus compared to CSA in the DIRECT study, pharmacokinetic studies to assess mycophenolic acid drug exposure were not performed. Whether calcineurin inhibitor to mTOR inhibitor class switch is beneficial in patients with BK viremia or BKN or both is a subject of ongoing clinical research. The effectiveness of currently available antiviral agents are of uncertain benefit. Lastly, the routine recommendations of fluoroquinolone prophylactic therapy in the post transplant period awaits results of randomized, double-blind, placebocontrolled trials.

The authors' suggested approach for post transplant screening and management of BKV-associated clinical syndromes are shown in figure 1. BKV-specific immune response and development of T-cell and antibody-based vaccines against BKV are subjects of future research.

\section{References}

1. Dharnidharka V, Cherikh W, Abbott KC (2009) An OPTN analysis of national registry data on treatment of BK virus allograft nephropathy in the United States. Transplantation 87: 1019-1026.

2. Brennan DC, Agha I, Bohl DL, Schnitzler MA, Hardinger K, et al. (2005) Incidence of BK with tacrolimus versus cyclosporine and impact of preemptive immunosuppression reduction. AmJ Transplant 5(3): 582-594.

3. Hirsch HH, Vincenti F, Friman S, Tuncer M, Citterio F, et al. (2012) Polyomavirus BK replication in De Novo kidney transplant patients receiving tacrolimus or cyclosporine: A prospective, randomized, multicenter study. Am J Transplant [Epub ahead of print].

4. Acott PD, O'Regan PA, Lee SH, Crocker JF (2008) In vitro effect of cyclosporine A on primary and chronic BK polyomavitrus infection in Vero E6 cells. Transp Infect Dis 10: 385-390.

5. Li YJ, Weng CH, Lai WC, Wu HH, Chen YC, et al. (2010) A suppressive effect of cyclosporine $\mathrm{A}$ on replication and noncoding region activation of polyomavirus BK virus. Transplantation 89: 299-306.

6. Hardinger KL, Koch MJ, BohI DJ, Storch GA, Brennan DC (2011) Polyomavirus BK (BKV) replication in renal tubular epithelial cells is inhibited by mTOR inhibitors but activated by tacrolimus in a pathway involving the FKBP12. Am J Transplant 2011, Abstract 497.

7. Hardinger KL, Koch MJ, Bohl DJ, Storchand GA, Brennan DC, et al. (2010) BK-virus and the impact of pre-emptive immunosuppression reduction: 5-year results. Am J Transplant 10: 407-415.

8. Suwelack B, Malyar V, Koch M, Sester M, Sommerer C (2012) The influence of immunosuppressive agents on BK virus risk following kidney transplantation, and implications for choice of regimen. Transplantation Rev 26: 201-211.

9. Araki K, Turner AP, Shaffer VO, Gangappa S, Keller SA, et al. (2009) mTOR regulates memory CD8 T-cell differentiation. Nature 460: 108-112.

10. Turner AP, Shaffer VO, Araki K, Martens C, Turner PL, et al. (2011) Sirolimus enhances the magnitude and quality of viral-specific CD8 T-cell responses to vaccinia virus vaccination in rhesus macaques. Am J Tranplant 5: 465-474.

11. Anyaegbu El, Almond PS, Milligan T, Allen WR, Gharaybeh S, et al. (2012) Intravenous immunoglobulin therapy in the treatment of BK viremia and nephroparhy in pediatric renal transplant recipients. Pediatr Transplant 16 : E19-E24.

12. Johnston O, Jaswal D, Gill JS, Doucette S, Fergusson DA, et al. (2010) Treatment of polyomvirus infection in kidney transplant recipients: a systematic review. Transplantation 89: 1057-1070.

13. Randhawa PS, Schonder K, Shapiro R, Farasati N, Huang Y (2010) Polyomavirus BK neutralizing activity in human immunoglobulin preparations. Transplantation 89: 1462-1465.

14. Bohl DL, Brennan DC, Ryschkewitsch C, Gaudreault-Keener M, Major EO, et al. (2008) BK virus antibody titers and intensity of infections after renal transplantation. J ClinVirol 43: 184-189.

15. Schachtner T, Müller K, Stein M, Diezemann C, Sefrin A, Babel N, et al. (2011) BK virus-specific immunity kinetics: a predictor of recovery from polyomavirus BK-associated nephropathy. Am J Transplant 11: 2443-2452. 
Citation: Pham PT, Schaenman J, Pham PC (2012) BK Virus Screening and Management Following Kidney Transplantation: An Update. J Transplant Technol Res 2: e119. doi:10.4172/2161-0991.1000e119

16. Gabardi S, Waikar SS, Martin S, Roberts K, Chen J, et al. (2010) Evaluation of fluoroquinolones for the prevention of BK viremia after renal transplantation. Clin J Am SocNephrol 5: 1298-1304.

17. Mommeja Marin H, Marty F, Boeckh M, Winston D, Rowley S.D, et al. (2012) "CMX001 May Mitigate Signs of BK Virus (BKV) Associated Bladder and Kidney End-organ Damage" 24th International Congress of The Transplantation Society, Berlin, Germany.

18. Kasiske BL, Zeier MG, Chapman JR, Craig JC, Ekberg H, et al. (2010) KDIGO clinical practice guideline for the care of kidney transplant recipients: a summary. Kidney Int 77: 299-311.

19. Hirsch HH, Brennan DC, Drachenberg CB, Ginevri F, Gordon J, et al. (2005) Polyomavirus-associated nephropathy in renal transplantation: interdisciplinary analyses and recommendations. Transplantation 79: 1277-1286.
20. Schold JD, Rehman S, Kayle LK, Magliocca J, Srinivas TR, et al. (2009) Treatment for BK virus: incidence, risk factors, and outcomes for kidney transplant recipients in the United States. Transplant Int 22: 626-634.

21. Casadei DH, del C Rial M, Opelz G, Golberg JC, Argento JA, et al. (2001) A randomized and prospective study comparing treatment with high dose intravenous immunoglobulin with monoclonal antibodies for rescue of kidney grafts with steroid-resistant rejection. Transplantation 71: 53-58.

22. Luke PP, Scantlebury VP, Jordan ML, Vivas CA, Hakala TR, et al. (2001) Reversal of steroid- and anti-lymphocyte antibody-resistant rejection using intravenous immunoglobulin (IVIG) in renal transplant recipients. Transplantation 72: 419-422. 